\title{
Backward bifurcations in dengue transmission dynamics
}

\begin{abstract}
A deterministic model for the transmission dynamics of a strain of dengue disease, which allows transmission by exposed humans and mosquitoes, is developed and rigorously analysed. The model, consisting of seven mutually-exclusive compartments representing the human and vector dynamics, has a locallyasymptoticallymstable disease-free equilibrium (DFE) whenever a certain epidemiological threshold,known as the basic reproduction number ðR0P is less than unity. Further, the model exhibits the phenomenon of backward bifurcation, where the stable DFE coexists with a stable endemic equilibrium. The epidemiological consequence of this phenomenon is that the classical epidemiological requirement of making R0 less than unity is no longer sufficient, although necessary, for effectively controlling the spread of dengue in a community. The model is extended to incorporate an imperfect vaccine against the strain of dengue. Using the theory of centre manifold, the extended model is also shown to undergo backward bifurcation. In both the original and the extended models, it is shown, using Lyapunov function theory and LaSalle Invariance Principle, that the backward bifurcation phenomenon can be removed by substituting the associated standard incidence function with a mass action incidence. In other words, in addition to establishing the presence of backward bifurcation in models of dengue transmission, this study shows that the use of standard incidence in modelling dengue disease causes the backward bifurcation phenomenon of dengue disease.
\end{abstract}

Keyword: Dengue, Mosquitoes, Equilibria, Stability, Bifurcation, Vaccine 